\title{
„Viele Aspekte von Leitlinien beruhen häufig auf Erfahrungen in der klinischen Routine“"
}

Wie unterstützen Leitlinien Ärzte und Ärztinnen im klinischen Alltag, wie profitieren Patienten und Patientinnen von ihnen und wie sieht der Prozess bei der Entstehung von Leitlinien aus, gerade wenn sie international ausgerichtet sind? Darüber haben wir mit Prof. Dr. Dr. Mike P. Wattjes vom Institut für Diagnostische und Interventionelle Neuroradiologie der Medizinischen Hochschule Hannover gesprochen. Professor Wattjes ist verantwortlicher Erstautor der neuen internationalen Empfehlungen zur Bildgebung bei Multipler Sklerose (MS). Die Empfehlungen werden sowohl von den europäischen als auch von den nordamerikanischen MS-Organisationen unterstützt und sind in der renommierten Fachzeitschrift The Lancet Neurology veröffentlicht worden.

Professor Wattjes, Multiple Sklerose (MS) ist eine chronisch entzündliche Erkrankung des zentralen Nervensystems. Hierzulande sind rund 250000 Menschen betroffen. Welche Rolle spielen die Radiologie und die Magnetresonanztomografie bei der Diagnostik und Behandlung von MS?

Die Bildgebung durch die Magnetresonanztomografie (MRT, die Red.) bei der MS hat in den letzten Jahren stark an Bedeutung gewonnen. Früher war es so, dass die älteren Diagnosekriterien noch auf fast rein klinischen und laborchemischen Befunden beruhten. Mit der Verbesserung der MRT und besonders deren breiter Verfügbarkeit hat sie die Diagnose, aber auch die Verlaufsbeobachtung der MS quasi revolutioniert: Mit dem MRT kann man noch sensitiver vorgehen und Veränderungen im Gehirn und im Rückenmark darstellen, ohne dass der Patient klinische Symptome aufweist. Zum einen ermöglicht dies die
Diagnose einer MS zu einem frühen Zeitpunkt, das heißt bei einem erstmaligen neurologischen Schub. Zum anderen ist dies besonders für die Verlaufsbeobachtung wichtig, denn man möchte ja in der Therapie keine neue Krankheitsaktivität mehr sehen. Die Möglichkeiten des MRT geben dem Neurologen Hinweise, etwa ob die Medikamente, die der Patient beziehungsweise die Patientin einnimmt, effizient genug sind, oder ob die Behandlung verstärkt und zu einer noch effektiveren Behandlung übergegangen werden muss.

Sie haben nun gemeinsam mit Experten und Expertinnen aus verschiedenen Ländern neue internationale Leitlinien zur Bildgebung bei MS in der klinischen Routinepraxis erarbeitet. Wie wichtig ist es, dass Ärzte und Ärztinnen auch international und nicht nur in Deutschland oder Europa bei dieser Krankheit nach einheitlichen Standards arbeiten?

Es ist sehr wichtig, dass diese Standardisierung der MS-Bildgebung auf internationalen Füßen steht, denn wesentliche Fragen, die wir jetzt und in der Zukunft für MS-Patienten beantworten müssen, sind abhängig von großen Datensätzen und zwar von Datensätzen, die standardisiert und damit vergleichbar sind, also wesentliche Prädiktionsmodelle hinsichtlich der individuellen Krankheitsprogression erlauben. Prädiktionsmodelle kann man nur mit riesigen Datensätzen entwickeln. Das Gleiche gilt bei der Vorhersage von Behandlungserfolgen von einzelnen Medikamenten, die auch nur mit großen Datensätzen gelingen. Was den zukünftigen Einsatz automatisierter Bildauswertungsalgorithmen betrifft, sind wir auch von großen standardisierten Datensätzen abhängig. Diese sind nur möglich, wenn man sich medizinisch auf einen

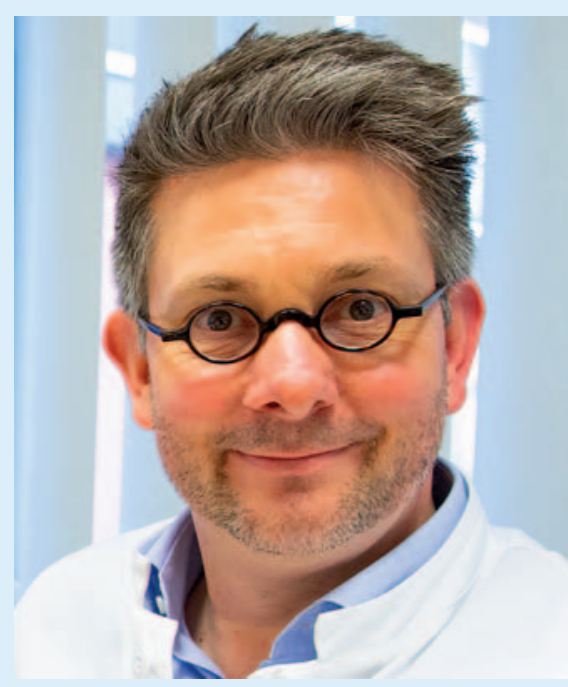

Prof. Dr. Dr. Mike P. Wattjes

gewissen Grundstandard einigt - national und international.

Sind Sie bei der Entwicklung der Leitlinien zur Multiplen Sklerose Vorreiter oder haben Sie bereits vorhandene weiterentwickelt?

Diese 2021-Leitlinien, die wir nun geschrieben haben, beruhen auf einer Weiterentwicklung von Leitlinien, die im Jahre 2015 und 2016 von unterschiedlichen Gruppen bereits vorgestellt worden sind. Diese Leitlinien sind natürlich ständig im Fluss, es gibt immer neue Entwicklungen bei MS, neue Medikamente kommen auf den Markt, die kontroverse Diskussion über den Einsatz der Gadolinium-basierten Kontrastmittel et cetera. Insofern gab es mehrere Entwicklungen, die uns dazu veranlasst haben, die bestehenden Leitlinien zu revidieren und aufzufrischen. Dazu kommt, dass es uns ein großes Anliegen war, diese unterschiedlichen Leitlinien aus Europa 
und Nordamerika zu einer großen globalen Leitlinie zu homogenisieren.

Wie lange hat der Prozess der Leitbilderarbeitung bei Ihnen und der Gruppe von Experten und Expertinnen, mit der Sie zusammengearbeitet haben, gedauert?

Ich habe immer zu meiner Frau gesagt, es hat zwei Schwangerschaften gedauert, also 18 Monate. Es war eine sehr arbeitsintensive und aufregende Zeit und gegen Ende dieses Prozesses waren wir alle ein bisschen erschöpft. Am Ende des Tages hilft einem nur das Wissen um die Verantwortung gegenüber den Patienten über diesen anstrengenden und zeitraubenden Prozess hinweg. Aber Leitlinien sind wichtig - nicht primär für uns persönlich, sondern in erster Linie für die Patienten, für die Medizinentwicklung seitens der Pharmaindustrie sowie für Neurologen und (Neuro-)Radiologen.

Multiple Sklerose ist zwar therapier-, aber nicht heilbar. Betroffene müssen ein häufiges Monitoring und Verlaufskontrollen durchführen lassen. Welche Empfehlungen geben Sie und Ihre Kollegen und Kolleginnen in den neuen Leitlinien etwa beim Thema Kontrastmittelgabe?

Dieses Thema war eine unserer Hauptmotivationen, um bestehende Leitlinien zu revidieren. Wir haben 2015 relativ unkritisch empfohlen, kontrastmittelverstärkte T1gewichtete Sequenzen als MRT Marker zu benutzen, um inflammatorische Krankheitsaktivitäten zu dokumentieren und damit auch zu untersuchen, ob eine medikamentöse Therapie effizient ist oder nicht. Doch wir haben nun die jüngste Diskussion um die Ablagerung von elementarem Gadolinium in der tiefen grauen Substanz zum Anlass genommen, diesen Aspekt zu revidieren. Wir sind jetzt der Meinung, dass man für viele Fragestellungen, insbesondere, was das Therapie-Monitoring betrifft, Kontrastmittel nicht mehr zwingend benötigt, weil wir eben mit anderen MRTParametern, mit den sogenannten aktiven T2-Läsionen, also mit neuen Entmarkungsherden, die auftreten, oder Entmarkungsherden, die 50 Prozent größer werden im Vergleich zur Referenzuntersuchung, einen guten alternativen Marker haben, ohne auf Kontrastmittel zurückgreifen zu müssen.
Selbstverständlich gibt es immer noch Indikationen, wo Kontrastmittel sinnvoll sind. Das gilt insbesondere für die Diagnose der MS. Aber für viele Indikationen, die die Verlaufsbeobachtung und das Therapie-Effizienz-Monitoring und das Sicherheits-Monitoring betreffen, sind Kontrastmittel nicht mehr zwingend notwendig und können eingespart werden.

Könnten Sie ganz generell beschreiben, wie die Diskussion und die Konsensfindung bei Leitlinien abläuft, auch international?

Wichtig sind bei diesem Prozess mehrere Schritte, wobei die Vorbereitung der Konsensfindung das Entscheidende ist. Bei uns war es so, dass wir zunächst ein Konzept erarbeitet haben, in dem die Themen aufgeführt wurden, die einer möglichen Revision bedürfen. Dieses Proposal haben wir dann an die Leitlinienkommission verschickt, sodass sich jeder auf diese Konsensus-Findung vorbereiten konnte. Dann wurden die identifizierten Themen in einem Konsensus-Meeting besprochen und soweit wie möglich evidenz-basiert diskutiert, Dieses Meeting hat 2019 in Graz stattgefunden. Entsprechend der Konsensus-Findung wurden alle diskutierten Aspekte dokumentiert.

Nach dem zweitägigen Konsensus-Meeting wurden die Ergebnisse in ein Manuskript eingearbeitet. Danach zirkulierte dieses Manuskript unter den beteiligten Mitgliedern der Leitlinienkommission. In dieser etwas längeren Zirkulations- und Manuskriptphase wurden auch noch weitere neue Aspekte, die erst nach dem KonsensusMeeting relevant wurden, in das Manuskript eingearbeitet.

Wenn man bedenkt, wie lange es dauern kann, bis Leitlinien erstellt sind und wie rasant sich die Medizin in manchen Bereichen entwickelt, könnte sich die Frage der Aktualität stellen. Wie schätzen Sie dieses Thema ein?

Zu lange darf ein solcher Prozess natürlich nicht dauern, weil dann neue Aspekte und Themen relevant werden, welche dann wieder berücksichtigt und eventuell eingearbeitet werden müssen. In unserem Fall war das nicht dramatisch, denn Innovationen auf dem Gebiet der Bildgebung bei der MS passieren nicht von einem Tag auf den anderen. Bis neue bildgebende Methoden so validiert sind, dass sie letztendlich auch Eingang in die klinische Praxis finden können, dauert es zum Teil mehrere Jahre. Bei anderen Leitlinien kann das aber anders aussehen. Insbesondere bei Therapieleitlinien kann beispielsweise die Zulassung eines neuen Medikaments die Therapielandschaft komplett verändern, sodass die entsprechenden Leitlinien umfänglich aktualisiert und angepasst werden müssen. Aber das war bei uns wie gesagt nicht der Fall.

Kritiker und Kritikerinnen monieren zuweilen, dass Leitlinien, auch wenn sie evidenzbasiert sind, von unzureichender methodischer Qualität sein können. Sie sehen darin die Gefahr, dass die in Leitlinien enthaltenen Empfehlungen bei ihrer Umsetzung nicht den gewünschten Effekt einer besseren Versorgung von Patienten und Patientinnen erreichen. Wie schätzen Sie diese Kritik ein?

Diese Kritik ist zum Teil berechtigt. Man kann Leitlinien häufig nicht ausschließlich evidenzbasiert ausrichten, weil es für viele Aspekte nicht genug Evidenz, zum Beispiel keine Klasse-1-Evidenz gibt, um sie darauf begründen zu können. Viele Aspekte von Leitlinien, und das schließt auch unsere Empfehlung mit ein, beruhen häufig auf langjährigen Erfahrungen in der klinischen Routine. Dies wird zum Teil kontrovers diskutiert. Kritik an bestehenden Leitlinien sehe ich persönlich nicht negativ, denn in dem Moment, wo es Kritik an Leitlinien gibt, entsteht auch ein Dialog. Einen kritischen Dialog erfahre oder interpretiere ich als fruchtbar und motivierend, um die Dinge noch weiter zu verbessern. Leitlinien sind grundsätzlich nicht der Goldstandard, sondern sie sollen Mediziner leiten und dabei unterstützen, in der klinischen Routine die richtigen Entscheidungen zu treffen. Sie sind aber nicht verpflichtend. Es gibt klinische Situationen in denen man sich begründet über eine Leitlinie hinwegsetzen kann.

In welchen medizinischen Fachgebieten sehen Sie aktuell einen besonderen Nutzen oder eine besondere Notwendigkeit von Leitlinien? 
Das sind Themen oder Fachgebiete, bei denen die zur Verfügung stehenden Daten für die Kollegen häufig nicht konklusiv und sehr schwierig zu interpretieren sind. Das gilt sowohl für radiologische beziehungsweise diagnostische Themen als auch für die
Therapie von bestimmten Erkrankungen. Ein gutes Beispiel ist die aktuelle und kürzlich veröffentlichte DGN-Leitlinie für MS. Die MS-Therapielandschaft wird immer breiter und heterogener, und auch da ist es deshalb wichtig, den Ärzten mithilfe von
Leitlinien Orientierung und Hilfestellung zu bieten, um so der bestmöglichen Therapieentscheidung den Weg zu bereiten, zum Wohle des individuellen Patienten. 